\title{
A simple way to obtain average occupation on quantum systems
}

\author{
Antonio Freitas Neto*1@, José Maria Villas-Bôas \\ ${ }^{1}$ Universidade Federal de Uberlândia, Instituto de Física, Uberlandia, MG, Brasil
}

Received on August 05, 2018; Revised on October 09 2018; Accepted on October 10, 2018.

\begin{abstract}
In this work, we present a simple method to obtain time average occupation of quantum systems with timeindependent Hamiltonian. Our method is fast and can be used for system with large Hilbert space. The average occupation can give insights and help finding the best parameter to be used in experiments or for a more complex simulation using time-depended Hamiltonian and density matrix. As an example of application, we use this method to find the best parameters to send a laser to excite a two-level system coupled to cavity mode to a final state.

Keywords: time evolution, average occupation, two-level, cavity, quantum mechanics
\end{abstract}

\section{Introdução}

The evolution of time-independent Hamiltonian can be easily evaluated by diagonalizing the Hamiltonian and writing the wave function as a sum of exponential terms. This can be done analytically for system with small Hilbert space and numerically for larger system, as described in many textbooks of quantum mechanics [1,2]. Once we have the time-dependent wave function we can easily obtain the expectation values and its time average by integrating it over long times. The time integration for system with larger Hilbert space usually takes a lot of computation resources as it requires a time integration over long time sequences. The average occupation of a quantum states can give good insight of the best parameters that an experimentalist needs to manipulate the system, or the best parameters needed to simulate the system when a time-dependent field is applied, and a density matrix is needed to solve. We have being using the average occupation in Refs. [ [5 9] ], usually doing the numerical integration which uses a lot of computation resources.

In this paper we derive an analytical expression for the time average occupation of a quantum state of timeindependent Hamiltonians. This analytical expression can be used for system with large Hilbert space, speeding up its numerical computation. As an example, we will use it to find the best parameters of an external field needed to excite an atom in a cavity. Here we will use an Jaynes-Cummings Hamiltonian [3, 4] with the addition of an extra term to describe an external pumping by a laser field, as in Ref. [ 9]].

*Endereço de correspondência: antonio.neto@ifms.edu.br.
To derive our analytical expression for the average occupation, let's begin with the time-dependent Schrödinger equation $(\hbar=1)$

$$
\frac{\partial|\psi\rangle}{\partial t}=-\imath H|\psi\rangle
$$

where $H$ is the Hamiltonian written in a matrix form in the bases of our observables, which we will call the original basis, and $|\psi\rangle$ is the vector representation of the wave function, also known as ket representation. Written in this form, we have a set of $N$ differential equations, where $N$ is the size of our Hilbert space. For time-independent Hamiltonian, the general solution for this equation, which give us the time evolution of our system, is

$$
|\psi(t)\rangle=\exp (-\imath H t)|\psi(0)\rangle
$$

where

$$
|\psi(0)\rangle=\sum_{i=1}^{N} a_{i}|i\rangle
$$

is the initial state written in the original basis. If the Hamiltonian is diagonal, we have the stationary states in the original basis. For non-diagonal Hamiltonian matrix, we can compute the exponential of the matrix by diagonalizing it. In this case, the time evolution of the wave function can be written as

$$
\begin{aligned}
|\psi(t)\rangle & =C^{-1} C \exp (-\imath H t) C^{-1} C|\psi(0)\rangle \\
& =C^{-1} \exp (-\imath \lambda t) C|\psi(0)\rangle,
\end{aligned}
$$

where $C$ and $C^{-1}$ are the matrix change of basis and its inverse, respectively, that diagonalize $H$, and $\lambda$ is 
a diagonal matrix with eigenvalues $\lambda_{j}$. Notice that in the expression above we have the multiplication of three matrix, which multiply the vector $|\psi(0)\rangle$. Notice also the $C|\psi(0)\rangle$ is just the representation of the initial state in the basis that diagonalize $H$, which we will call

$$
|\psi(0)\rangle_{\lambda}=C|\psi(0)\rangle=\sum_{j=0}^{N} b_{j}\left|\lambda_{j}\right\rangle
$$

where

$$
b_{j}=\sum_{i=1}^{N} C_{j i} a_{i},
$$

being $C_{j i}$ the matrix elements of the change basis matrix. The time evolution in the basis that diagonalize $H$ is then written as

$$
|\psi(t)\rangle_{\lambda}=\sum_{j=0}^{N} \exp \left(-\imath \lambda_{j} t\right) b_{j}\left|\lambda_{j}\right\rangle
$$

and in the original basis

$$
|\psi(t)\rangle=C^{-1}|\psi(t)\rangle_{\lambda}=D|\psi(t)\rangle_{\lambda},
$$

with $D=C^{-1}$ being the inverse matrix of the matrix $C$, used just to simplify the notation. By doing the matrix multiplication, we can write

$$
|\psi(t)\rangle=\sum_{i, j} D_{i j} b_{j} \exp \left(-\imath \lambda_{j} t\right)|i\rangle
$$

where $D_{i j}$ is the matrix elements of $D$. As an example, for a simple two-level system, the Hilbert space size is $N=2$ and we have

$$
\begin{aligned}
|\psi(t)\rangle= & {\left[D_{00} b_{0} e^{-\imath \lambda_{0} t}+D_{01} b_{1} e^{-\imath \lambda_{1} t}\right]|0\rangle } \\
& +\left[D_{10} b_{0} e^{-\imath \lambda_{0} t}+D_{11} b_{1} e^{-\imath \lambda_{1} t}\right]|1\rangle,
\end{aligned}
$$

which we find in many quantum mechanics text books.

Now that we have the time-dependent wave-function we can compute the probability to find the system in any state $|i\rangle$ of the original basis by

$$
P_{i}(t)=|\langle i \mid \psi(t)\rangle|^{2},
$$

which can be written as

$$
\begin{aligned}
P_{i}(t) & =\sum_{j=1}^{N}\left|D_{i j} b_{j}\right|^{2} \\
& +2 \sum_{j, k} D_{i j} b_{j} D_{i k} b_{k} \cos \left[\left(\lambda_{j}-\lambda_{k}\right) t\right] .
\end{aligned}
$$

Here we have used that for a Hermitian matrix the eigenvectors are real, so the changing basis matrix are also real. Finally, we want to obtain the average occupation of the quantum state in the original basis, which we will define as

$$
\overline{P_{i}}=\frac{1}{t_{f}} \int_{0}^{t_{f}} P_{i}(t) d t
$$

This integral can be easily done, as the only time-dependence is the cosine function. In the limit of $t_{f} \rightarrow \infty$,

$$
\lim _{t_{f} \rightarrow \infty} \frac{\sin \left[\left(\lambda_{j}-\lambda_{k}\right) t_{f}\right]}{t_{f}}=0,
$$

for any $j, k$. This allow us to write the average occupation as

$$
\overline{P_{0}}=\sum_{j=1}^{N}\left|D_{i j} b_{j}\right|^{2}
$$

This analytical solution only depends on the initial state component of the original basis $\left(a_{i}\right)$, the change basis matrix $C$ and its inverse $D$, which can be obtained by diagonalize the Hamiltonian $H$ and take the eigenstates coefficients to build the change basis matrix $C$ and evaluate its inverse $D$.

To exemplify this result, let assume the Jaynes-Cummings Hamiltonian describing a two-level system with frequency $\omega_{0}$ inside a cavity with mode frequency $\omega_{c}$, with the addition of an external pumping with frequency $\omega_{p}$ of the two-level system. An schematic representation of our system can be seen in Fig. 1, and its Hamiltonian, using the Rotation Wave Approximation (RWA) [10] can be written as $(\hbar=1)$

$$
\begin{aligned}
H= & \omega_{0} \sigma_{+} \sigma_{-}+\omega_{c} a^{\dagger} a+g\left(\sigma_{+} a+\sigma_{-} a^{\dagger}\right) \\
& +\frac{\Omega(t)}{2}\left(\sigma_{+} e^{-\imath \omega_{p}}+\sigma_{-} e^{\imath \omega_{p}}\right)
\end{aligned}
$$

where $\sigma_{ \pm}$are the pseudospin operators for the two-level system, $a^{\dagger}$ and $a$ are the creation and annihilation operators for photons inside the cavity, $g$ is the coupling between the cavity field and the two-level system, and $\Omega(t)$ is the interaction strength of the external field with the two-level system.

Assuming $\Omega(t)$ constant and using a unitary transformation, the time dependence of the Hamiltonian can be removed, and we can write it as

$$
\begin{aligned}
H= & \left(\delta_{c}-\delta_{p}\right) \sigma_{+} \sigma_{-}+\delta_{p} a^{\dagger} a \\
& +g\left(\sigma_{+} a+\sigma_{-} a^{\dagger}\right)+\frac{\Omega}{2}\left(\sigma_{+}+\sigma_{-}\right),
\end{aligned}
$$

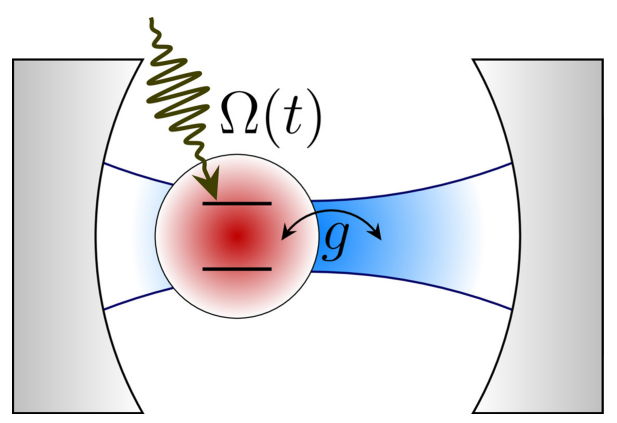

Figure 1: Schematic representation of a two-level system inside a cavity under coherent pumping. 
where $\delta_{c}=\omega_{0}-\omega_{c}$ and $\delta_{p}=\omega_{c}-\omega_{p}$ are the detuning between the two-level system and the cavity and the detuning between the cavity and the laser frequency, respectively.

Once we have a time-independent Hamiltonian we can use our average occupation approach to find a set of parameters that allow us, for example, to excite the system to very specific state final state. The advantage of our approach is that it allows us to vary different parameters in a large range with a low computer cost. In Fig. 2 we plot the average occupation probability for the ground state of our system, as a function of the lasercavity detuning $\left(\delta_{p}\right)$ and two-level-cavity detuning $\left(\delta_{c}\right)$, assuming that we pump the system with an constant coherent laser with $\Omega=1 g$ and that we started the system in the ground state with no photons in the cavity, $\psi(0)=|0,0\rangle$, where we use a basis $|s, n\rangle=|s\rangle \otimes|n\rangle$, with $|s\rangle$ being the two-level states and $|n\rangle$ the Fock state basis. Here we cut the Fock basis with $n_{\max }=5$. The false color code represents the average occupation of the initial state. From this plot we can see that in bright regions the system stays in the ground state, while in dark areas the laser is able to populate some states in the system, which we can easily identify by just plotting the average occupation of the other states, result not shown.

To find the exact parameters, we can make a cut in the 2D color plot. This can be seen in Fig. 3 where we plot the average occupation of all states, considering vertical cut at $\delta_{c}=-5 g$, as a function of $\delta_{p}$. This is equivalent to keep fixed cavity and two-level system frequency and vary the laser frequency. As we can see, the ground state gets depopulated when other states are getting populated. In the inset we amplify one of the regions where we see this behavior. In this particular case, the state being populated is the state $|1,1\rangle$ and the parameter where this occurs is $\delta_{p}=-2.775 \mathrm{~g}$.

With this set of parameters $\left(\delta_{c}=-0.5 g\right.$ e $\delta_{p}=$ $-2.775 \mathrm{~g}$ ) we now simulate the time evolution of the system considering as initial state the ground state of the two-level system with no photon in the cavity $|\psi(0)\rangle=$

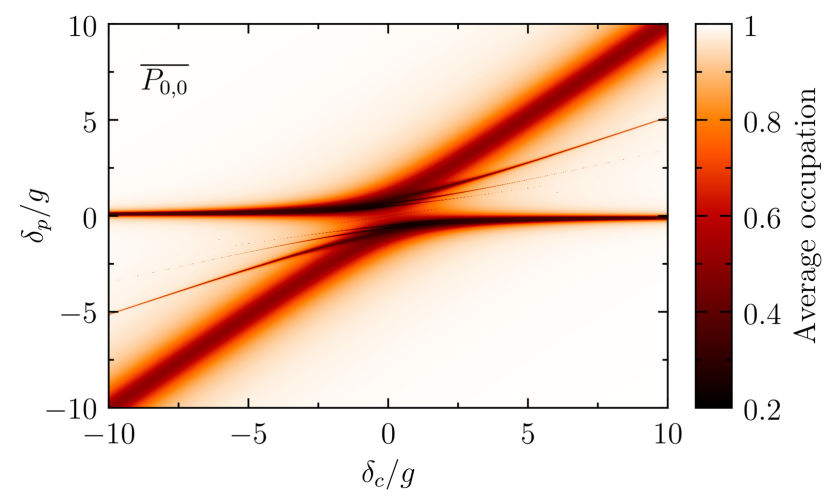

Figure 2: False color plot of the average occupation of the ground state $\overline{P_{0,0}}$ as function of the laser-cavity detuning $\left(\delta_{p}\right)$ and two-level-cavity detuning $\left(\delta_{c}\right)$.

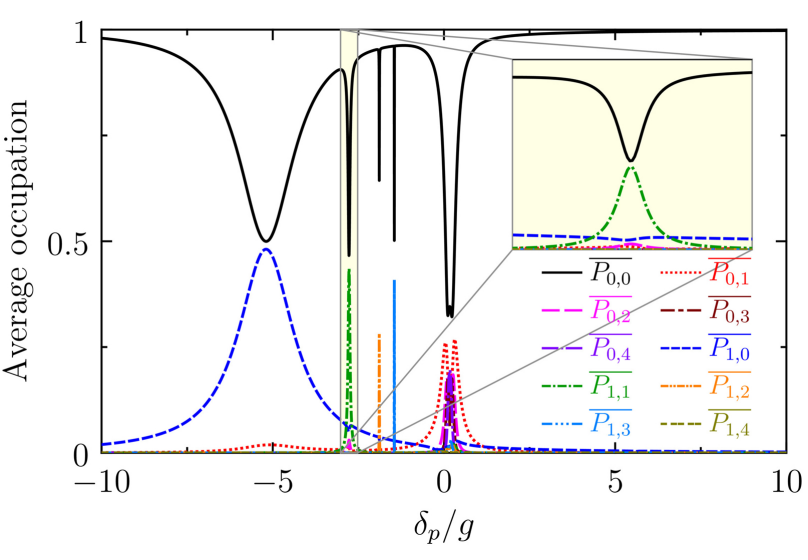

Figure 3: Average occupation of all states, considering vertical cut at $\delta_{c}=-5 g$ in Fig. 22 as a function of $\delta_{p}$. Inset shows an amplification around $\delta_{p}=-2.775 g$ where state $|1,1\rangle$ is populated.

$|0,0\rangle$ and a laser field with intensity $\Omega=1 g$. The results show that we have Rabi oscillations between the states $|0,0\rangle$ and $|1,1\rangle$ as seen in Fig. 4 which is a two-photon process, since the system present two excitation, one for the two-level and other for the photon in the Fock state. This was predicted in the average occupation as seen in Fig. 3 It would be very difficult to find the exactly parameter need for that evolution without using our average occupation approach.

In summary, in this paper we have presented a simple way to compute the average occupation of a timeindependent Hamiltonian. Our approach allows us to obtain an analytical expression that can be easily implemented using standard matrix diagonalization method. This speed-up the computation and allows to obtain the average occupation for systems with large Hilbert space and find suitable parameter for a more complex simulation using density matrix or for experimental investigations.

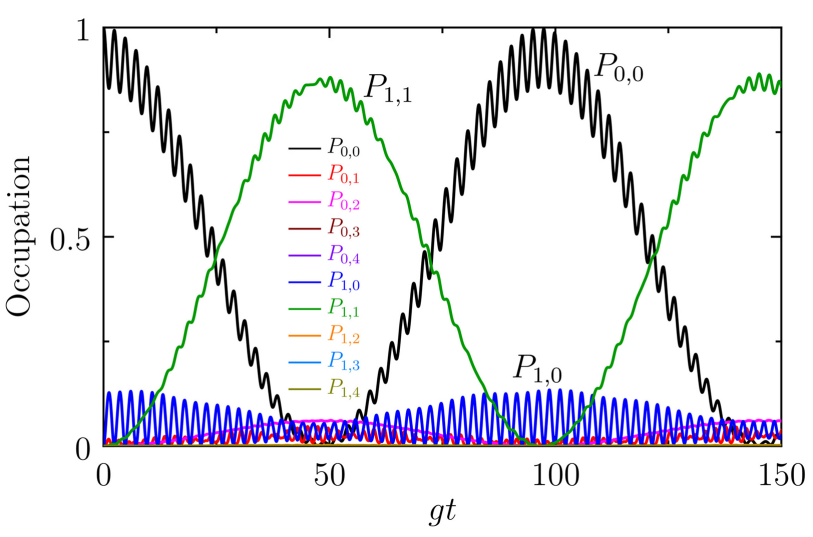

Figure 4: Time evolution of the system using the parameter obtained from the analysis of the average occupation, $\delta_{c}=$ $-0.5 g, \delta_{p}=-2.775 g$ and $\Omega=1 g$. 
We would like to acknowledge CAPES, CNPq and FAPEMIG by the financial support.

\section{References}

[1] C. Cohen-Tannoudji, B. Diu and F. Laloe, Quantum Mechanics (WWiley-VCH, Weinheim, 1991).

[2] J.J. Sakurai, Modern Quantum Mechanics (Addison Wesley, Boston, 1994).

[3] E.T. Jaynes and F.W. Cummings, Proceedings of the IEEE 51, 89, 1963.

[4] M.O. Scully and M.S. Zubairy, Quantum Optics (Cambridge University Press, Cambridge, 1997).

[5] J.M. Villas-Bôas, A.O. Govorov and S.E. Ulloa, Phys. Rev. B 69, 125342, 2004.

[6] J.M. Villas-Bôas, S.E. Ulloa and A.O. Govorov, PPhys. Rev. Lett. 94, 057404, 2005.

[7] J.M. Villas-Bôas, S.E. Ulloa and A.O. Govorov, Phys. Rev. B 75, 155334, 2007.

[8] H.S. Borges, L. Sanz, J.M. Villas-Bôas and A.M. Alcalde, Phys. Rev. B 81, 075322, 2010.

[9] A. Freitas, L. Sanz and J.M. Villas-Bôas, Phys. Rev. B 95, 2017.

[10] P.L. Knight and L. Allen, Phys. Rev. A 7 368, 1973. 